\title{
Three-dimensional Distribution of Grain Structure and Image Analysis
}

\author{
Atsumasa OKADA, Hiroshi KATSULAl and Mitsunori OKA \\ Faculty of Engineering, Chiba Institute of Technology, Tsudanuma, Narashino, Chiba-ken, 275 Japan.
}

(Received on November 30, 1989; accepted in the final form on March 9, 1990)

\begin{abstract}
The aim of this research is to establish a stereological method to characterize three-dimensional (3-D) distribution of grains in a metal from its sectional images with an application of the current image processing techniques. First of all, 3-D models of metals consisting of homogeneous grains and duplex grains were generated by simulating the processes of nucleation and crystallization with various nucleus distributions and growth rates. The frequency distribution of cutlengths of the ASTM or JSPS standard chart of austenite (No. 7 or 8) obtained by cutting them with equiinterval parallel lines comes into a normal distribution. It was confirmed that there is no significant difference between the standard chart and the homogeneous grain model by statistical tests, and relations among mean cut-length, grain size number, mean diameter and mean volume were derived on the homogeneous grain. For duplex grains, formulae were derived to calculate the degree of duplication and the fraction of volume occupied by different sizes of grains from the distribution of duplex grains.

The results can practically be utilized to obtain 3-D information about homogeneous and duplex grain structures with image processing techniques.
\end{abstract}

KEY WORDS: grain size; duplex grain; three-dimensional measurement; simulation; image processing.

\section{Introduction}

The process of grain structure generation is divided into two stages of nucleation and growth of crystals; grains grow around nuclei and grain boundaries are formed where grown grains meet with each other. The 3-D structure of a metallic crystal cannot be characterized with such techniques as computed tomography (CT) and magnetic resonance imaging (MRI), and has only been estimated from 2-D sectional images.

Image processing techniques have recently been introduced to the measurement of grain size distribution, ${ }^{1)}$ and have made it possible to quickly perform the measurements with higher precision. For general particles, several researches have been made to obtain the particle size distribution from sectional images, ${ }^{2-4)}$ and for metallic grains, the construction of grain structure models has also been attempted by varying the rates of nucleation and growth of crystals. ${ }^{5-7)}$

To estimate stereologically grain structures with an application of the current image processing techniques, the authors produce in a computer 3-D models consisting of homogeneous grains and duplex grains by altering the nucleus distribution and growth rates. That is, for the model of homogeneous grains, nuclei are uniformly located at random in the object space, and grains are grown equi-axially at a constant growth rate just as in the former methods. ${ }^{5-7)}$ Moreover, in order to make more real grain boundaries in a metallic model, a statistical normal distribution is assumed for the distribution of the growth rates of nuclei. For models of duplex grain structure consisting of two different sizes of grains, nuclei are non-uniformly located in an object space, and grains are grown equi-axially at normally distributed rates.

It is known that the cut-length distributions on the American Society for Testing and Material (ASTM) and the Japan Society for the Promotion of Science (JSPS) standard charts Nos. 7 and 8 are statistical normal distributions. ${ }^{8-10}$ ) In this paper, the difference of significance is statistically tested between the cut-length distribution for the homogeneous grain model and the ASTM or the JSPS standard charts, and the relationship is examined between 2-D measured values and 3-D informations for the constructed model.

In addition, it is attempted to construct a practical image processing system which can derive 3-D information from 2-D sectional images with an application of this method.

\section{Crystallization of Grain Structure by Gom- puter Simulation}

\subsection{Number of Grains Required for a Model}

A 3-D grain structure in a metal was generated in an ACOS 850-10 computer.

The number of grains required for statistical research with the relative error below $5 \%$ is likely to be more than 250 on a section of grain structure as shown in the following calculation.

If the permissible error in number of samples is represented by $L$, the standard deviation of a population by $\sigma$, and the number of grains by $n$,

$$
n=4 \sigma^{2} / L^{2},
$$


where $L$ is assumed to be $5 \%$ of the mean value $m$ of the population,

$$
n=4 \sigma^{2} /(0.05 m)^{2} \text {. }
$$

The value of $\sigma / m$ was estimated to be nearly 0.4 by measuring the ASTM and JSPS standard charts, independently of grain size number. Therefore,

$$
n=256 \text {. }
$$

From this, the ASTM and JSPS standard charts of higher grain size numbers than No. 7 have many grains enough to be treated statistically as shown in Table 1.

\subsection{Homogeneous Grain Model}

We created a 3-D model of homogeneous grain structure by computer simulation. The object space was divided into 20 by 20 by 20 cubes so as to obtain a sufficient number of grains, that is, more than 250 grains required statistically on a section of the model. In nucleation, one nucleus was sited at random in each cube of the object space, and then one grain was equi-axially grown from each nucleus. The growth rates were assumed to be constant, as in former investigations. ${ }^{5-7)}$ An enlargement of a part of the homogeneous grain model thus produced is presented in Fig. 1(a), where grain boundaries can be found to be linear. Then, assuming that growth rates obey a statistical normal distribution, we produced a homogeneous grain model by assigning the growth rates to grain nuclei at random. An enlargement of a part of that model is presented in Fig. 1(b), where grain boundaries can be found to be closer to real ones.

\subsection{Duplex Grain Model}

We produced 3-D models of duplex grain structure which consists of two different sizes of grains. The object space was divided into 40 by 40 by 40 cubes. In the process of nucleation, one nucleus was located at random in each cube for the fine grain portion of the object space and in each group of several cubes for the coarse grain portion. Grains were grown equi-axially from all nuclei with velocities obeying a normal distribution. Figs. 1(c) and 1(d) show sectional images of duplex grain models where fine grains

\begin{tabular}{|c|c|c|c|c|c|c|}
\hline & & Grain size No. & 5 & 6 & 7 & 8 \\
\hline \multirow{4}{*}{ Sort } & \multirow{2}{*}{ ASTM standard chart } & Numbre of grains & 85 & 203 & 480 & 964 \\
\hline & & Significance level $(\%)$ & 8.7 & 5.7 & 3.7 & 2.6 \\
\hline & \multirow{2}{*}{ JSPS standard chart } & Number of grains & 103 & 224 & 456 & 898 \\
\hline & & Significance level $(\%)$ & 7.9 & 5.3 & 3.7 & 2.7 \\
\hline
\end{tabular}

Table 1. Number of grains and significance level (\%) of standard charts.

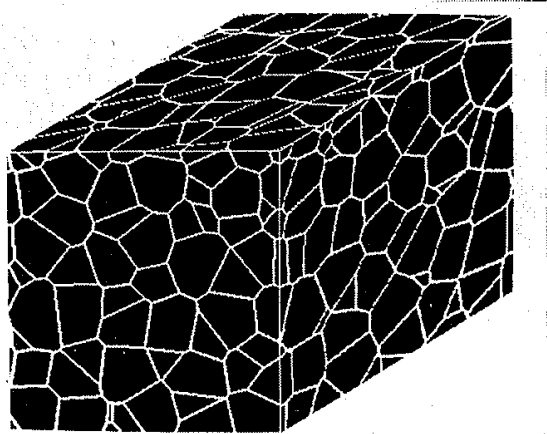

(a)

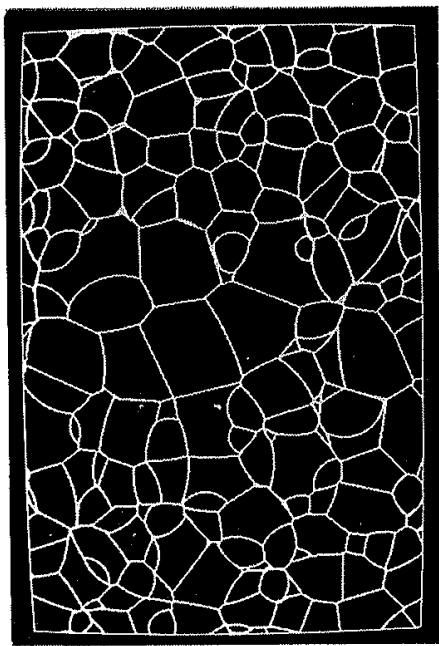

(c)

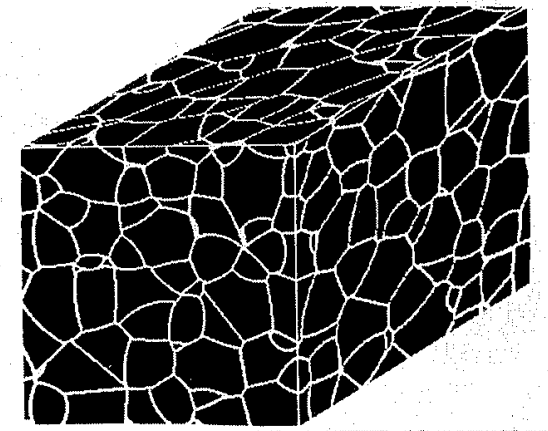

(b)

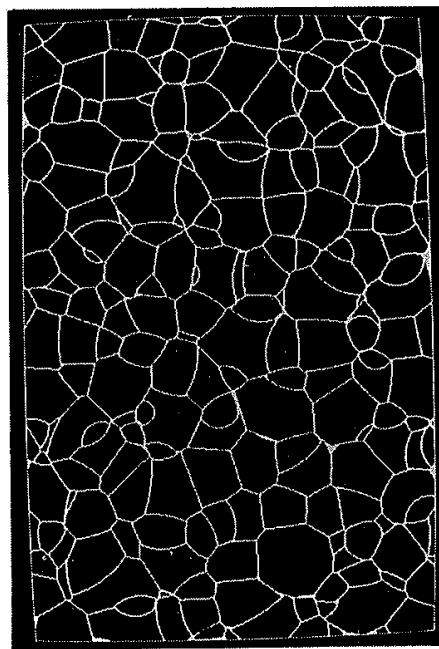

(d) (a) 3-D homogeneous grain model (Crystallization velocity is constant.)

(b) 3-D homogeneous grain model (Crystallization velocities obey a normal distribution.)

(c) Section of duplex grain model-1

(d) Section of duplex grain model-2

Fig. 1.

Images of homogeneous and duplex grain models... - - 
and coarse grains are mixed at various fixed volumetric ratios.

\section{Distribution of Three-dimensional Model}

\subsection{Distribution of Homogeneous Grain}

\subsubsection{Gut-length Distribution of Homogeneous} Grain

A section of grain structure was linearly scanned at a fixed interval so that one grain is passed through by one scanning line, and cut-lengths for each grain were measured. Since a normal probability chart has a vertical axis so graduated that the cumulative frequency curve of a normal distribution appears as a straight line, the normality of a given distribution can be examined by inspecting whether a given cumulative curve appears on a chart as a straight line or not. The cumulative frequency curves of cutlength distributions obtained from the sectioned homogeneous grain model and the ASTM standard charts of Nos. 8 and 7 approximated a straight line as shown in Figs. 2 and 3, respectively. Almost similar results were obtained from the JSPS standard chart of Nos. 8 and 7. These distributions can be considered to be normal. Furthermore, in this paper, the normality of the distributions was also examined by calculating skewness and peakedness of the distributions. As a result of it, it turned out that the cut-length

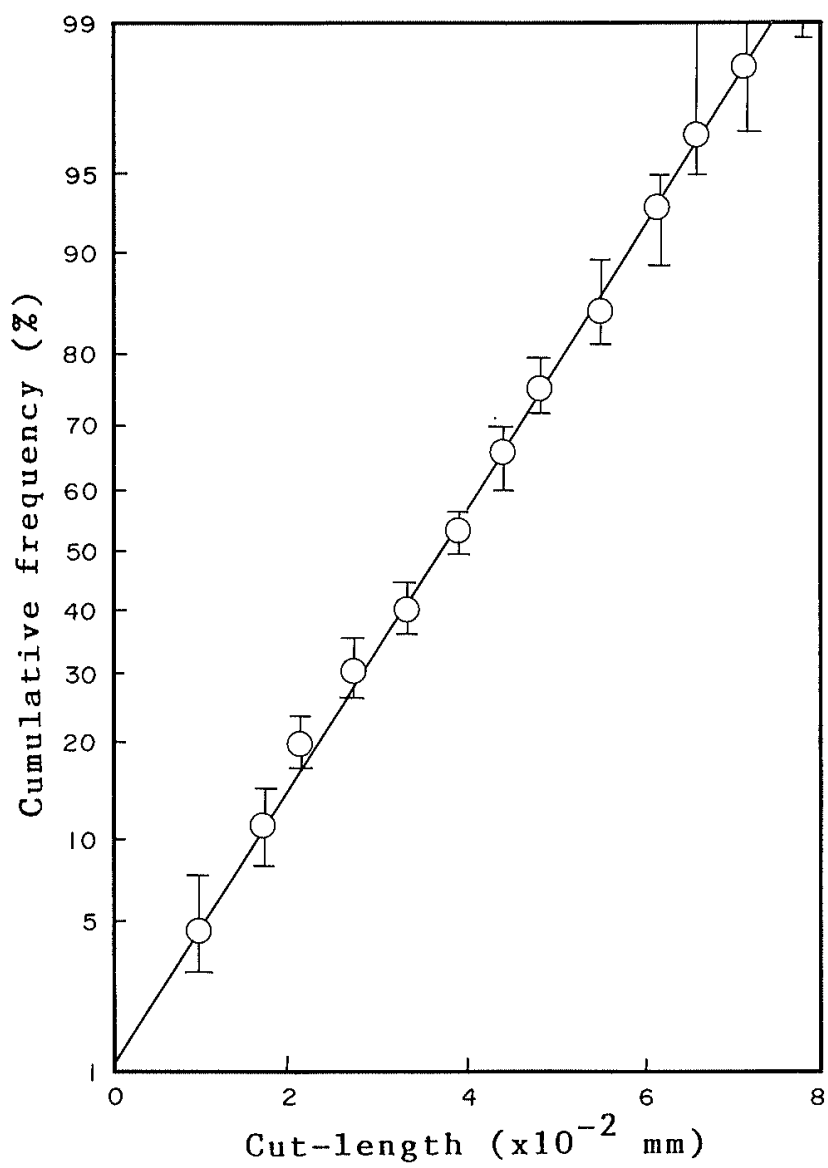

Fig. 2. Cumulative frequency curve of cut-length distributions on sectional images of the homogeneous grain model. distributions of grains of the sectioned homogeneous model and the standard charts are normal at $5 \%$ significant level.

3.1.2. Diameter Distribution of Homogeneous Grain

The distribution of grain diameters observed on a sectional image was measured, and cumulative frequency distributions of diameters on sections are shown on a normal probability chart in Fig. 4.

To estimate the distribution of grain diameters, maximum sectional diameters of each grain were measured. Fig. 5 shows a histogram of the distribution of grain diameters in the simulation model, and the cumulative frequency distribution is shown on a normal probability chart in Fig. 6.

\subsection{Measurement of Homogeneous Grain}

\subsubsection{Grain Size Number}

The relation of Eq. (4) was presented as a straight line in Fig. 7, and marks ( $O$ and $x$ ) in the figure, obtained from the ASTM and the JSPS standard charts were seen to be very close to the straight line. So it was recognized that the grain size number can be determined practically from the mean cut-length by the straight line in Fig. 7.

\subsubsection{Mean Diameter and Mean Volume}

The sectional area $A$ of a spherical grain having circular diameter $d_{0}$ is represented by,

$$
A=\pi\left(d_{0} / 2\right)^{2} \text {. }
$$

Let $l_{0}$ denote the mean value of the cut-lengths intersecting the circumference of the circular area,

$$
d_{0}=A / l_{0} .
$$

Substituting Eq. (3) into Eq. (2),

$$
l_{0}=\sqrt{\pi / 4 \cdot A}
$$

Let $G$ denote the grain size number of the JSPS standard,

$$
\begin{array}{ll} 
& A=(1 / 2)^{a+3} . \\
\text { Hence, } & l_{0}=\sqrt{\pi / 4 \cdot(1 / 2)^{G+3}}
\end{array}
$$

Substituting Eq. (2) into Eq. (3), the relation between $l_{0}$ and $d_{0}$ is,

$$
d_{0}=4 / \pi \cdot l_{0} \text {. }
$$

For the correction coefficient $K_{1}$ for Eq. (5), grain diameter $d$ is,

$$
d=K_{1}\left(4 / \pi \cdot l_{0}\right)
$$

From measurements on sectioned grains of the model,

$$
K_{1}=1.04
$$

For the diameter $D_{0}$ and volume $V_{0}$ of a spherical particle,

$$
\begin{aligned}
V_{0} & =4 / 3 \cdot \pi \cdot\left(D_{0} / 2\right)^{3} . \\
& =A \cdot D_{0} .
\end{aligned}
$$

Therefore,

$$
A=2 \pi / 3 \cdot\left(D_{0} / 2\right)^{2}
$$




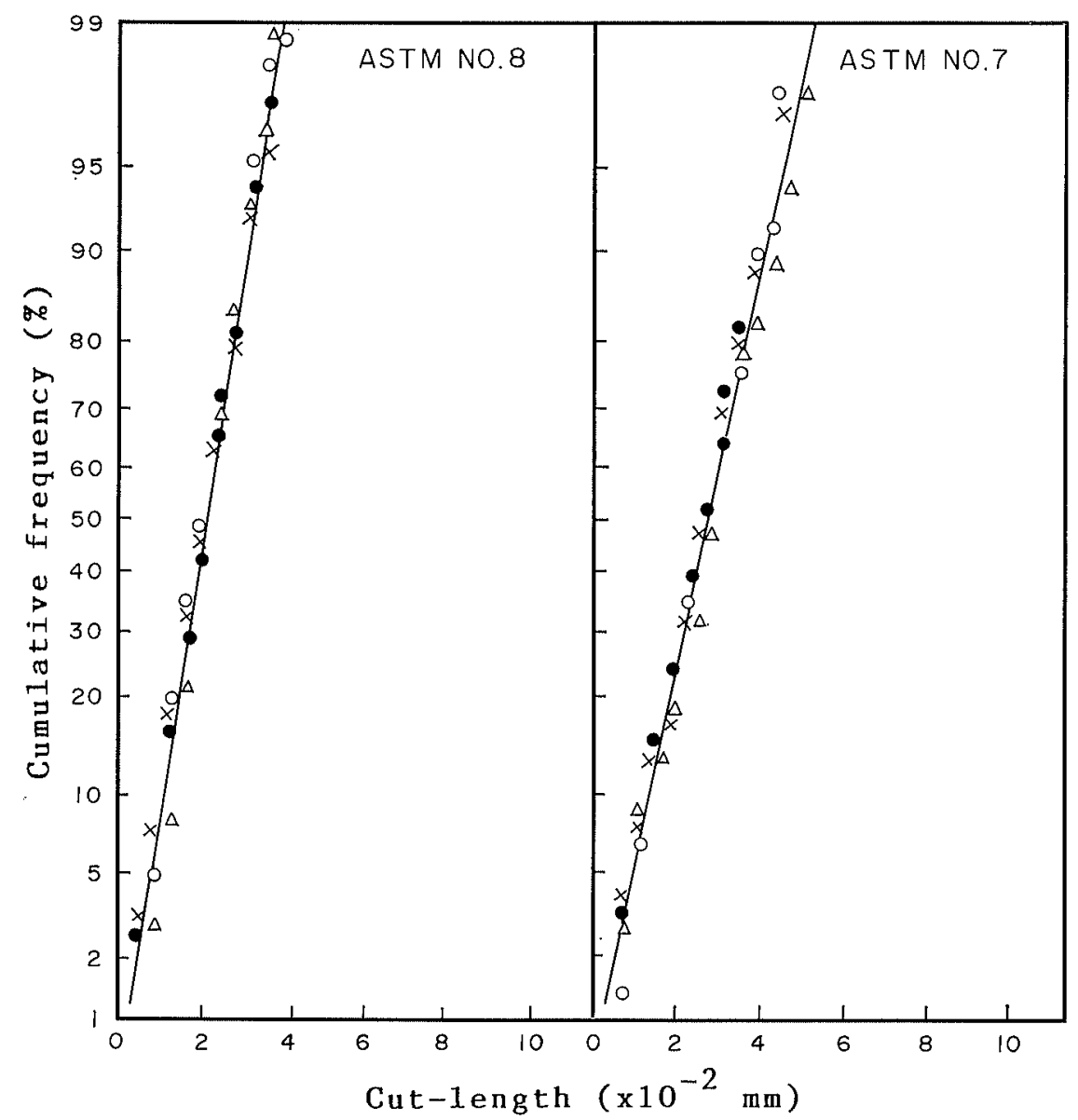

Fig. 3.

Cumulative frequency curve of cutlength distributions of the ASTM standard charts.
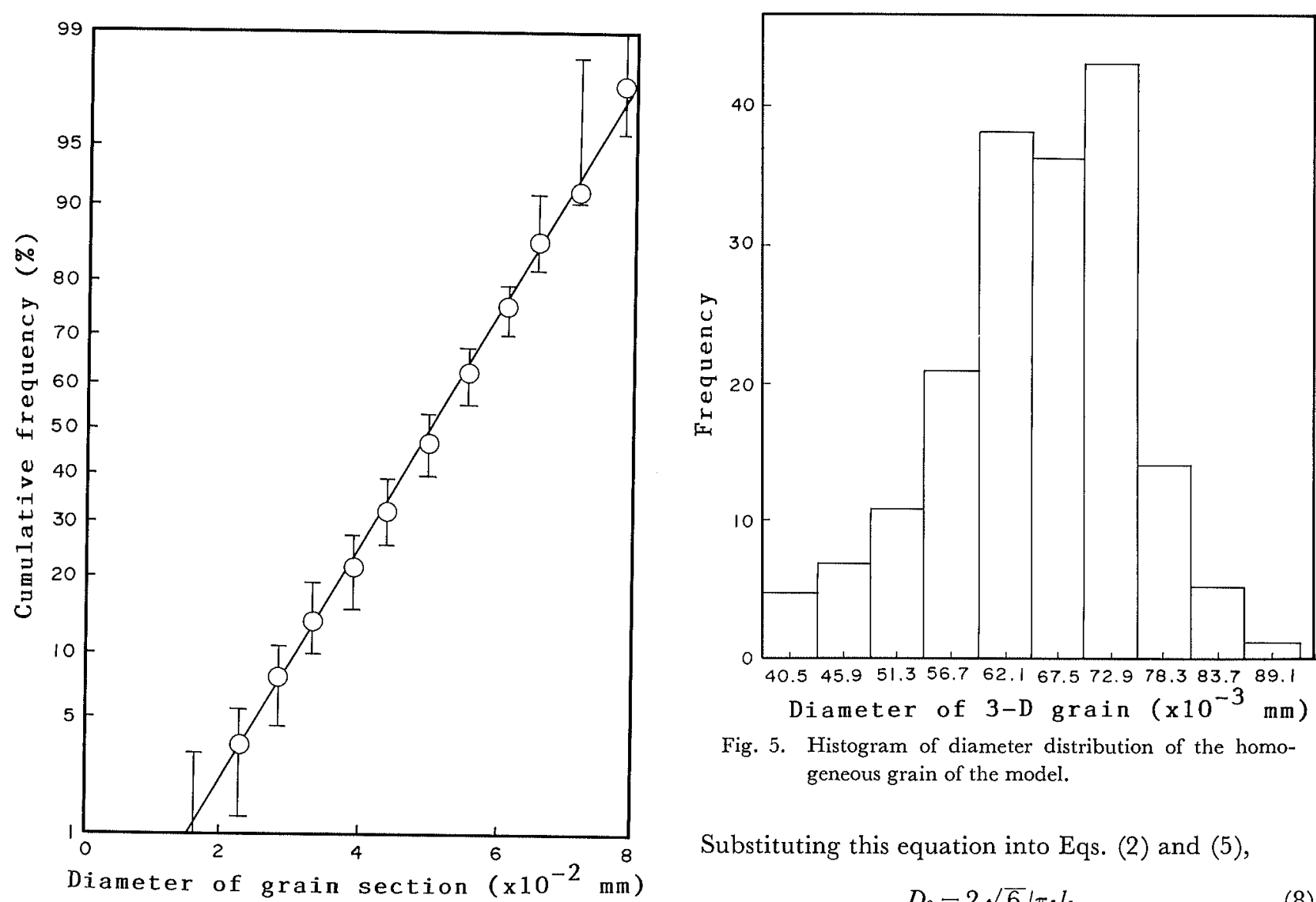

Fig. 5. Histogram of diameter distribution of the homogeneous grain of the model.

Substituting this equation into Eqs. (2) and (5),

$$
D_{0}=2 \sqrt{6} / \pi \cdot l_{0} .
$$

Fig. 4. Cumulative frequency curve of diameter distributions on sectional images of the homogeneous grain.

Due to Eqs. (7) and (8),-therelation between $l_{0}$ and 
$V_{0}$ is,

$$
V_{0}=8 \sqrt{6} \cdot l_{0}^{3} / \pi^{2}
$$

For the correction coefficient $K_{2}$ for Eq. (8), 3-D diameter $D$ is,

$$
D=K_{2}\left(2 \sqrt{6} / \pi \cdot l_{0}\right)
$$

From the measurements on the 3-D homogeneous grain model,

$$
K_{2}=1.09
$$

From the correction coefficient $K_{3}$ for Eq. (9), $V$ of 3-D grain is,

$$
V=K_{3}\left(8 \sqrt{6} \cdot l_{0}^{3} / \pi^{2}\right)
$$

From the measurements on the 3-D homogeneous grain model,

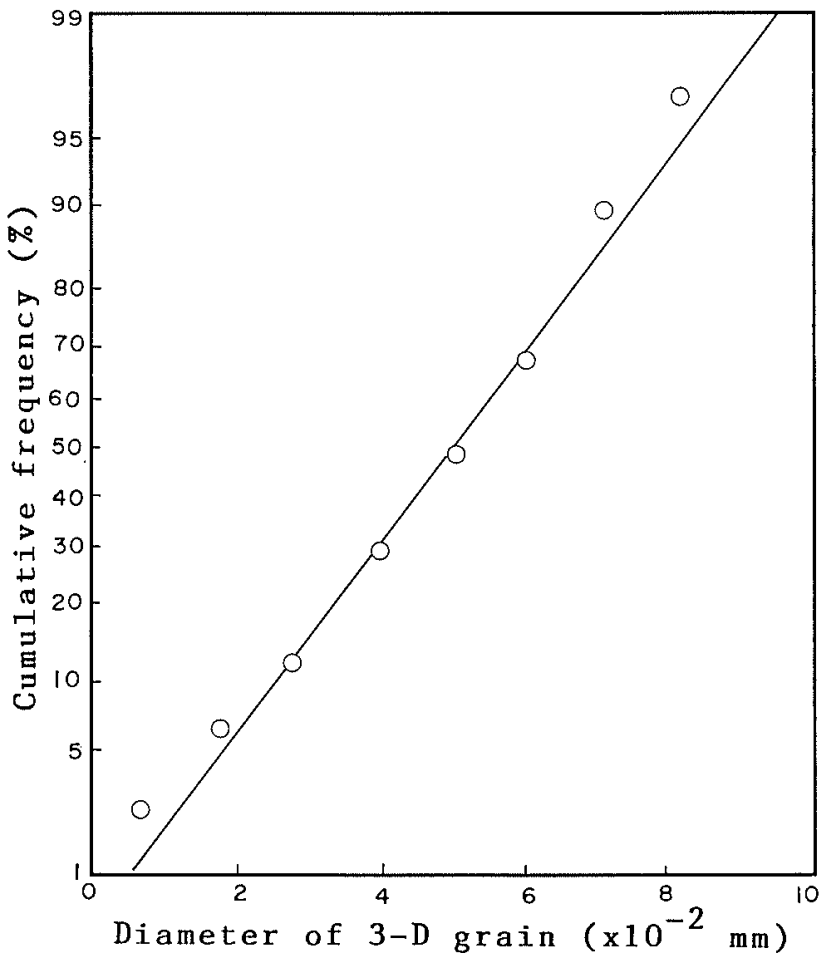

Fig. 6. Cumulative frequency curve of diameter distribution of the homogeneous grain model.

$$
\kappa_{3}=1.30 \text {. }
$$

Due to Eqs. (10) and (11), the mean diameter and mean volume of a solid grains can be estimated respectively by the mean cut-length obtained from the measurement on a sectioned surface of a metallic substance.

\subsection{Measurement of Duplex Grains}

3.3.1. Cut-length Distribution of Duplex Grains

If the grain size numbers of 2 kinds of grains contained in a duplex grain structure are denoted by $G_{f}$ and $G_{c}$, the numbers of the two grains contained in a unit area by $\mathcal{N}_{f}$ and $\mathcal{N}_{c}$, and the numbers of segments along one scanning line in a unit area by $n_{f}$ and $n_{c}$, respectively,

and

$$
\begin{aligned}
\mathcal{N}_{f} & =2^{a_{f+3}}, \\
\mathcal{N}_{c} & =2^{a_{c+3}}, \\
n_{f} & =\sqrt{\mathcal{N}_{f}}=\sqrt{2^{a_{f+3}}},
\end{aligned}
$$$$
n_{c}=\sqrt{\mathcal{N}_{c}}=\sqrt{2^{a_{c+3}}} \text {. }
$$

Let a denote the ratio of the occupied areas of 2 kinds of different sizes of grains on a sectioned duplex grain structure, and let $n$ the number of segments along a given scanning line, then

$$
n=a n_{f}+(1-a) n_{c}=a \sqrt{2^{G_{f+3}}}+(1-a) \sqrt{2^{G_{c+3}}} .
$$

The distribution $f_{i}$ of cut-lengths of duplex grains is represented by distributions $f_{f}$ and $f_{c}$ for 2 kinds of homogeneous grains as,

$$
f_{i}=a f_{f}+(1-a) f_{c} .
$$

\subsubsection{Degree of Duplication}

The mean value $m_{0}$ of the cut-length distribution can be represented by a homogeneous grain size number $G_{o}$ by Eq. (4),

$$
m_{0}=0.88 \sqrt{(1 / 2)^{G_{0+3}}} .
$$

If the standard deviation of the cut-length distribution is denoted by $S_{i}$, and the grain size number by $G_{i}$ corresponding to $m_{0}+S_{i}$,
$m_{0}:$ Mean cut-length
$G$ : Grain size number
$O$ : JSPS standard chart
$x$ : ASTM standard chart

Fig. 7.

Relation between grain size number and mean cut-length.

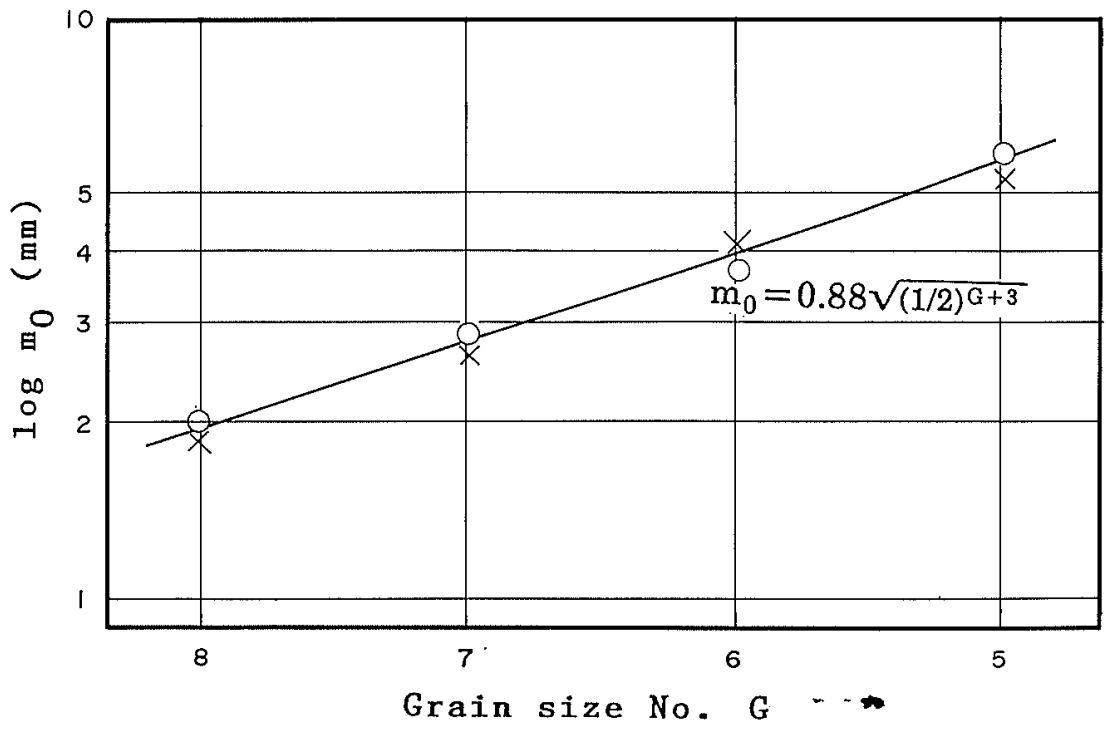




$$
m_{0}+S_{i}=0.88 \sqrt{(1 / 2)^{G_{i+3}}} .
$$

If the standard deviation $S$ is represented by degree of duplication $\sigma_{S}$,

$$
\sigma_{S}=G_{o}-G_{i} .
$$

Due to Eqs. (12) and (13),

$$
\sigma_{S}=\log \left(1+S_{i} / m_{0}\right) / \log 1.4 .
$$

Generally, the mean value $m$ and the standard deviation $S$ in statistically classified data are given as follows,

$$
\text { and } \quad S=\sqrt{\sum\left(x_{i}-m\right)^{2} \cdot f_{i} / n} \text {. }
$$

Therefore,

$$
\sigma_{S}=\log \left\{1+\sqrt{n \sum\left(x_{i}-m\right)^{2} \cdot f_{i}} / \sum x_{i} f_{i}\right\} / \log 1.4 .
$$

Hence,

$$
\begin{aligned}
\sigma_{S} & =\log [1 \\
& +\sqrt{\left\{a n_{f}+(1-a) n_{c}\right\} \sum\left(x_{i}-m\right)^{2} \cdot\left\{a f_{f}+(1-a) f_{c}\right\}} / \\
& \left.\sum x_{i} \cdot\left\{a f_{i}+(1-a) f_{c}\right\}\right] / \log 1.4 . \quad \ldots \ldots \ldots \ldots \ldots \ldots \ldots \ldots \ldots \ldots \ldots \ldots
\end{aligned}
$$

\subsubsection{Degree of Mixture}

For a homogeneous grain structure,

$$
a=1 \text {, }
$$

therefore, Eq. (14) is reduced to,

$$
\sigma_{S}=\log \left(1+S_{i} / m_{0}\right) / \log 1.4 \text {. }
$$

Since the value of $S_{i} / m_{0}$ is already known to be 0.4 in Sec. 2.1, the degree of duplication for homogeneous grain structure must be

$$
\sigma_{S}=\log 1.4 / \log 1.4=1 .
$$

For instance, the degree of duplication for the ASTM standard chart is shown in Fig. 8. Since $\sigma_{7}$ is located on the same coordinate with $m_{6}$ in the figure, it is seen that the relation $\sigma_{S}=1$ holds.

The relation between degree of duplication $\sigma_{S}$ and fraction of area a can be calculated from $\mathrm{Eq}$. (14) and the result is shown in Fig. 9 for various parameter

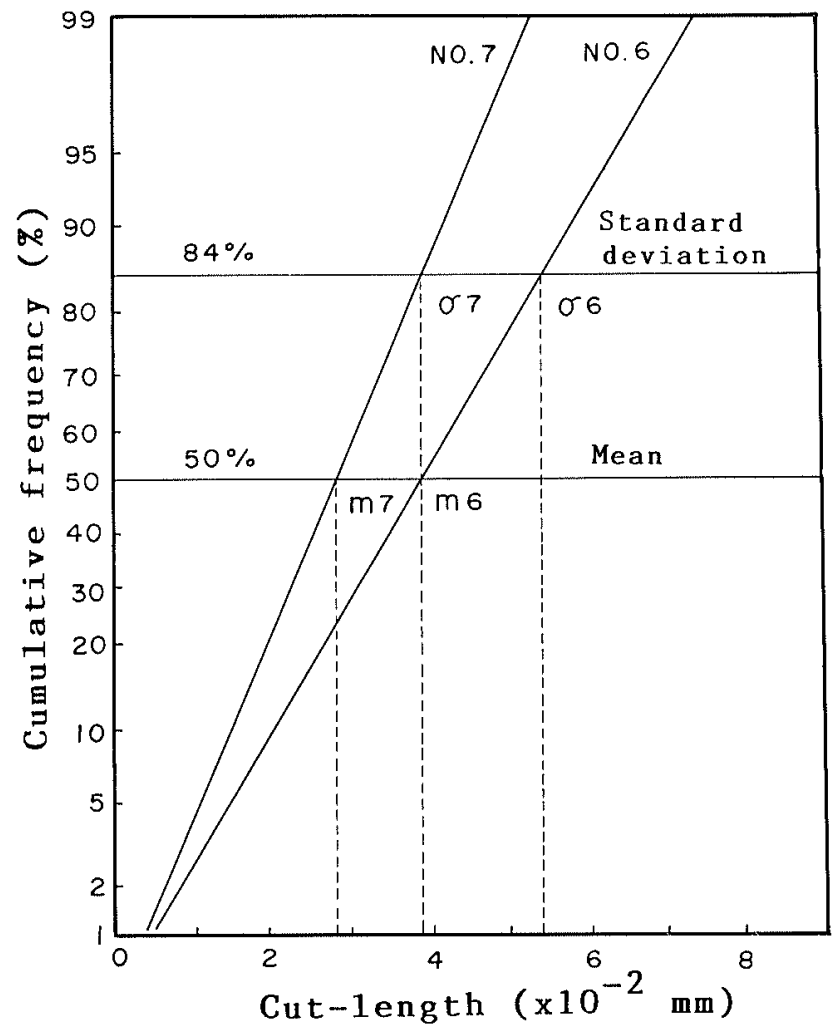

Fig. 8. Relation between mean cut-length and standard deviation.

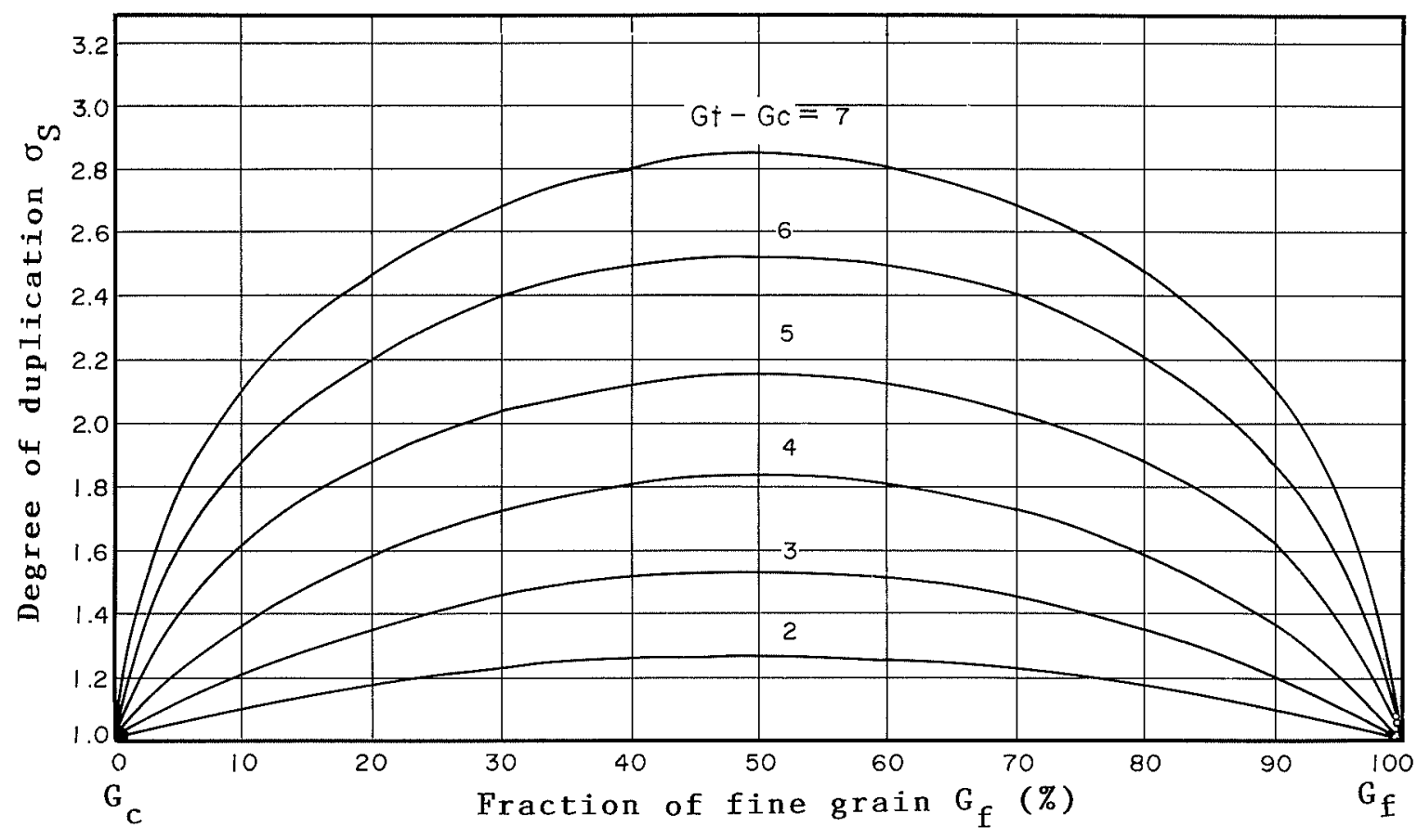

Fig. 9. Relation between degree of duplication $\sigma_{S}$ and fraction of fine grains in duplex grains. 
values of $G_{f}$ and $G_{c}$. Conversely, using this figure, the area fraction for a given duplex grain structure can be determined from the cut-length distribution and the cumulative frequency curve for the structure.

\section{Application of Image Analysis}

\subsection{Image Analyzing System}

The measuring method described above was applied to an existing image analyzing system. The block diagram of the image analyzing system depicted in Fig. 10 consists of an image input device, a fundamental structure and an output device. The image input device takes an object image through a microscope and a TV camera. An image from a TV camera is A/D converted and transferred into a $640 \times$ $512 \times 8$ bit storage. Then each image is transformed into binary one, and the following parameter values are output from the system:

1) Gain size number $G$ for mean cut-length $l_{0}$

2) Mean grain diameter $d$ for mean cut-length $l_{0}$

3) Spherical grain diameter $D_{0}$ for mean cut-length $l_{0}$

4) Spherical grain volume $V_{0}$ for mean cut-length $l_{0}$

5) Degree of duplication $\sigma_{S}$ for standard deviation $S$

6) Mixture ratio $a(\%)$ obtained by $G_{f}-G_{e}$ and by the relation shown in Fig. 9.

\subsection{Examples of Determination of Grain Size and Degree of Mixture}

Two different sizes of grains are usually included in a metal, which is called duplex grain. A 3-D model of a metal was generated and the distributions were measured on that model. The distribution curve for duplex grain structure did not present a straight line on a probability chart as shown in Fig. 11. The grain size number of each grain in the duplex grain was determined from the comparison with the straight lines representing homogeneous grains on a probability chart. Consequently, it has been confirmed that the degree of duplication $\sigma_{S}$ can be calculated from the standard deviation $\sigma$, and the ratio of grain mixture can be obtained from the difference of grain size numbers $G_{f}-G_{c}$ as mentioned in Sec. 3.3.3.

\section{Conclusion}

(1) In this research, we constructed two kinds of 3-D models of metals consisting of homogeneous grains and duplex grains, and showed that in particular the cut-length distribution for the homogeneous grain model can be looked upon as a normal distribution, as well as that for the ASTM and JSPS standard charts. Moreover, we demostrated that for the duplex grain model, the cumulative curve of the cutlength distribution distinctly deviates from a straight

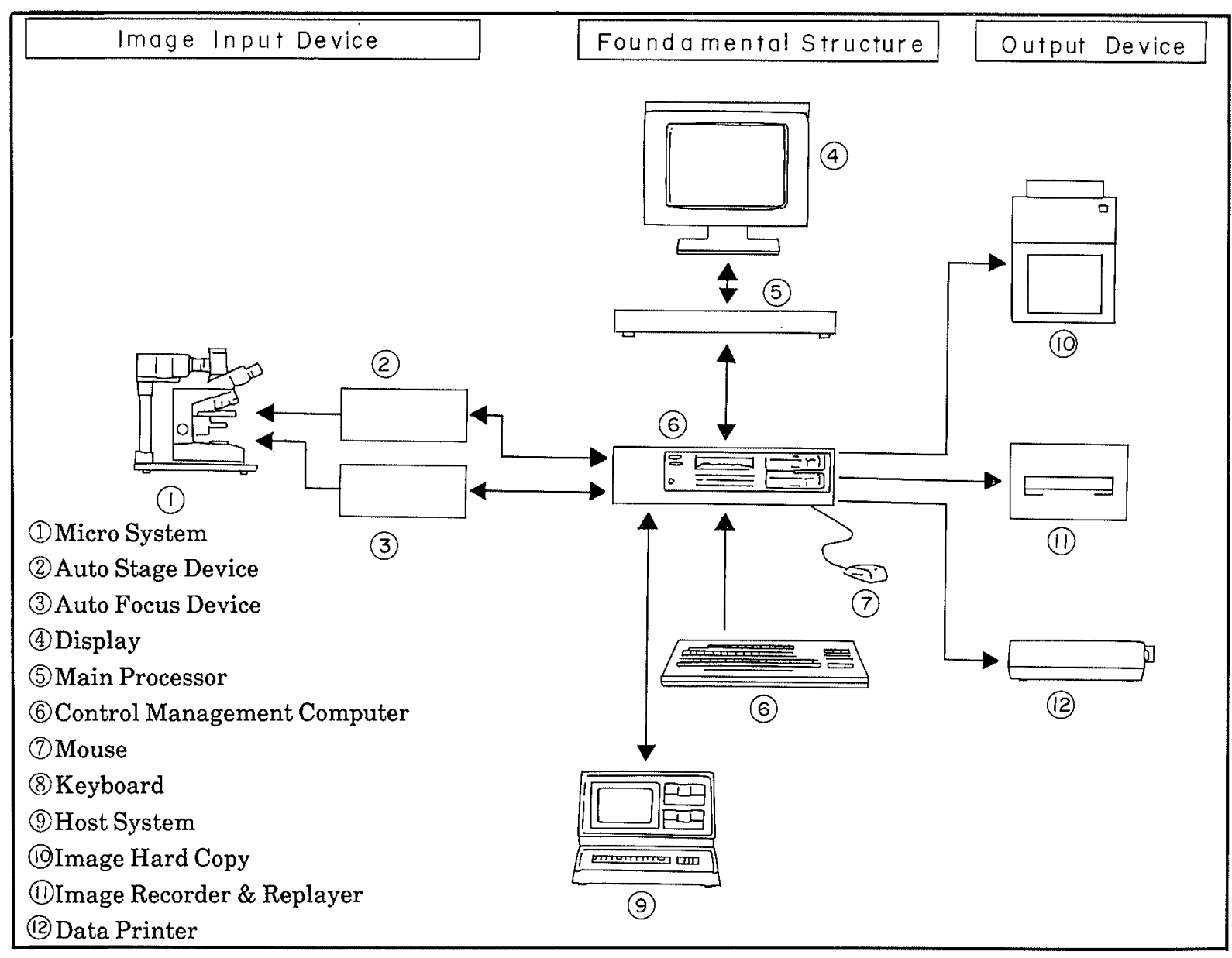

Fig. 10. Block diagram of an image analyzing system. 


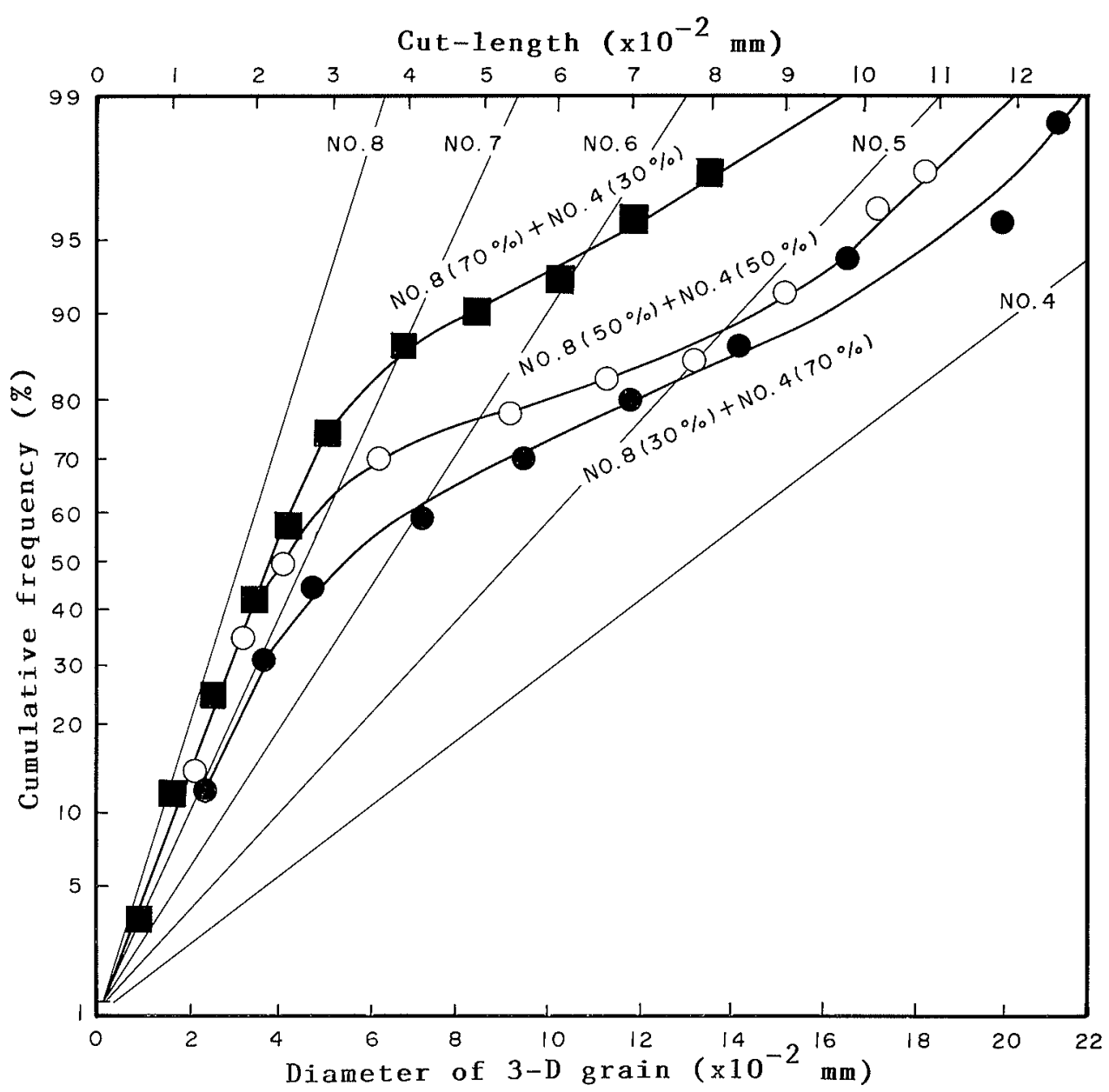

Fig. 11. Cumulative frequency distribution of duplex grain model.

line on a probability chart, which shows a characteristic of the duplex grain metal.

(2) Since the cut-length distribution on a sectioned homogeneous grain metal may be looked upon as a normal distribution, relations were derived to estimate the mean grain diameter and mean volume from the mean of the normal distribution, and then the formulae representing the relationship among the mean cut-length, the mean grain diameter and the mean volume were confirmed for the 3-D homogeneous grain model. For the duplex grain model, the degree of duplication was obtained from the cumulative curve on the probability chart, and the mean and standard deviation for the distribution. Then the formulae to calculate the grain size number and the mixture ratio were derived. Lastly for the duplex models consisting of two different sizes and various mixture ratios of grains, the formulae which expresses the relationship among the mean and standard deviation of the cut-length distribution, the degree of duplication, the grain size numbers, and the mixture ratio were derived.

(3) Application of the results described above to a practical image processing system makes it possible to obtain such 3-D information as grain diameter and volume distributions from sectional images of metallic structure as an aggregate of grains.

It may be expected that it is possible to estimate in three-dimensions the process of crystallization in metallic structures by comparing them with various kinds of crystal structures constructed by simulation.

\section{REFERENCES}

1) T. Takeuchi: Mater. Proc., 1 (1988), 541.

2) P. L. Goldsmith: Brit. J. Appl. Phys., 18 (1967), 813.

3) M. Belanger and W. D. Seufert: Biophysik, 9 (1972), 39.

4) R. P. King: Powder Tech., 21 (1978), 147.

5) W. A. Johnson and R. F. Mehl: Trans. Am. Inst. Min. Metall. Pet. Eng., 135 (1939), 416.

6) K.W. Mahin, K. Hanson and J. W. Morris, Jr.: Acta metall., 28 (1980), 443.

7) F. Mori and I. Miura: Tetsu-to-Hagané, 70 (1984), 1864.

8) A. Okada: Tetsu-to-Hagané, 48 (1962), 544.

9) S. Tanaka: The 19th Comm. (Steelmaking), the Japan Society for the Promotion of Science (JSPS), Rep. No. 7329 (Oct., 1963).

10) The Sub-committee for Measuring Austenite Grain Size in Steel, JSPS: Standard Method of Measuring the Austenite Grain Size in Steel, JSPS, Tokyo, (Nov. 4, 1965), 17. 